\title{
RADIATION EFFECTS ON MHD FLOW PAST AN IMPULSIVELY STARTED INFINITE VERTICAL PLATE WITH MASS DIFFUSION
}

\author{
P. CHANDRAKALA* \\ Department of Mathematics \\ Bharathi Women's College No. 34/2 \\ Ramanujam Garden Street \\ Pattalam, Chennai - 600 012, INDIA \\ E-mail: pckala05@yahoo.com \\ P. NARAYANA BHASKAR \\ CPCL, Chennai - 600 068, INDIA
}

\begin{abstract}
The effects of thermal radiation on a flow past an impulsively started infinite vertical plate in the presence of a magnetic field have been studied. The fluid considered is a gray, absorbing-emitting radiation but non-scattering medium. The dimensionless governing equations are solved by an efficient, more accurate, unconditionally stable and fast converging implicit scheme. The effects of velocity and temperature for different parameters such as the thermal radiation, magnetic field, Schmidt number, thermal Grashof number and mass Grashof number are studied. It is observed that the velocity decreases in the presence of thermal radiation or a magnetic field.
\end{abstract}

Key words: radiation, magnetic field, vertical plate, finite-difference.

\section{Introduction}

Processes involving coupled heat and mass transfer occur frequently in nature. They occur not only due to temperature difference, but also due to concentration difference or the combination of these two. The influence of the magnetic field on a viscous incompressible flow of electrically conducting fluid has its importance in many applications such as extrusion of plastics in the manufacture of rayon and nylon, purification of crude oil, pulp, paper industry, textile industry and in different geophysical cases, etc. In many process industries, the cooling of threads or sheets of some polymer materials is of importance in the production line. The rate of cooling can be controlled effectively to achieve final products of desired characteristics by drawing threads, etc. in the presence of an electrically conducting fluid subject to a magnetic field.

Radiative convective flows are encountered in countless industrial and environment processes, e.g., heating and cooling chambers, fossil fuel combustion energy processes, evaporation from large open water reservoirs, astrophysical flows, solar power technology and space vehicle re-entry. Radiative heat and mass transfer play an important role in manufacturing industries for the design of reliable equipment. Nuclear power plants, gas turbines and various propulsion devices for aircrafts, missiles, satellites and space vehicles are examples of such engineering applications.

England and Emery (1969) studied the thermal radiation effects of an optically thin gray gas bounded by a stationary vertical plate. Soundalgekar and Takhar (1993) considered the radiative free convective flow of an optically thin gray-gas past a semi-infinite vertical plate. Radiations effect on mixed

\footnotetext{
* To whom correspondence should be addressed
} 
convection along an isothermal vertical plate were studied by Hossain and Takhar (1996). In all above studies, a stationary vertical plate is considered. Raptis and Perdikis (1999) studied the effects of thermal radiation and a free convection flow past a moving infinite vertical plate. Again, Raptis and Perdikis (2003) studied thermal radiation effects on a moving infinite vertical plate in the presence of mass diffusion. The dimensionless governing equations were solved by the Laplace transform technique.

A boundary layer flow on moving horizontal surfaces was studied by Sakiadis (1961). Kumari and Nath (1999) studied the development of the asymmetric flow of a viscous, electrically conducting fluid in the forward stagnation point region of a two-dimensional body and over a stretching surface with an applied magnetic field, when the external stream or the stretching surface was set into impulsive motion from the rest. The effects of a transversely applied magnetic field on the flow of an electrically conducting fluid past an impulsively started infinite isothermal vertical plate were studied by Soundalgekar (1979). The dimensionless governing equations were solved using the Laplace transform technique.

The problem of an unsteady natural convection flow past an impulsively started infinite vertical plate with uniform heat and mass diffusion in the presence of thermal radiation and a magnetic field has not received attention of any researcher. Hence, the present study is to investigate the MHD flow past an impulsively started infinite vertical plate with thermal radiation by an implicit finite-difference scheme of CrankNicolson type.

\section{Basic equations and analysis}

A transient, laminar, unsteady natural convection flow of a viscous incompressible fluid past an impulsively started infinite vertical plate has been considered. It is assumed that the concentration $C^{\prime}$ of the diffusing species in the binary mixture is large in comparison to the other chemical species which are present.

Here, the $x$-axis is taken along the plate in the vertically upward direction and the $y$-axis is taken normal to the plate. Initially, it is assumed that the plate and the fluid are of the same temperature and concentration. The plate starts moving impulsively in the vertical direction with constant velocity $u_{0}$ and the temperature of the plate and the concentration level near the plate are also raised to $T_{w}^{\prime}$ and $C_{w}^{\prime}$. They are maintained at the same level for all time at time $t^{\prime}>0$. A transverse magnetic field of uniform strength $B_{0}$ is assumed to be applied normal to the plate. The induced magnetic field and viscous dissipation are assumed to be negligible. Then, under the usual Boussinesq's approximation, the unsteady flow is governed by the following equations

$$
\begin{aligned}
& \frac{\partial u}{\partial t^{\prime}}=g \beta\left(T^{\prime}-T^{\prime}\right)_{\infty}+g \beta^{*}\left(C^{\prime}-C_{\infty}^{\prime}\right)+v \frac{\partial^{2} u^{\prime}}{\partial y^{2}}-\frac{\sigma B_{0}^{2}}{\rho} u \\
& \rho C_{p} \frac{\partial T^{\prime}}{\partial t^{\prime}}=\frac{\partial^{2} T^{\prime}}{\partial y^{2}}-\frac{\partial q_{r}}{\partial y} \\
& \frac{\partial C^{\prime}}{\partial t^{\prime}}=D \frac{\partial^{2} C^{\prime}}{\partial y^{2}}
\end{aligned}
$$

The initial and boundary conditions are 


$$
\begin{aligned}
& t^{\prime}: u=0, \quad T^{\prime}=T_{\infty}^{\prime}, \quad C^{\prime}=C_{\infty}^{\prime}, \\
& t^{\prime}>0: u=u_{0}, \quad T^{\prime}=T_{w}^{\prime}, \quad C^{\prime}=C_{w}^{\prime} \quad \text { at } \quad y=0, \\
& u=0, \quad T^{\prime}=T_{\infty}^{\prime}, \quad C^{\prime}=C_{\infty}^{\prime} \quad \text { at } \quad x=0, \\
& u \rightarrow 0, \quad T^{\prime} \rightarrow T_{\infty}^{\prime}, \quad C^{\prime} \rightarrow C_{\infty}^{\prime} \quad \text { as } \quad y \rightarrow \infty .
\end{aligned}
$$

For the case of an optically thin gray gas the local radiant absorption is expressed by

$$
\frac{\partial q_{r}}{\partial y}=-4 a^{*} \sigma\left(T_{\infty}^{\prime 4}-T^{\prime 4}\right)
$$

We assume that the temperature differences within the flow are sufficiently small such that $T^{4}$ may be expressed as a linear function of the temperature. This is accomplished by expanding $T^{4}$ in a Taylor series about $T_{\infty}$ and neglecting higher-order terms, thus

$$
T^{\prime 4} \cong 4 T_{\infty}^{\prime 3} T^{\prime}-3 T_{\infty}^{\prime 4}
$$

By using Eqs (2.5) and (2.6), Eq.(2.2) reduces to

$$
\rho C_{p} \frac{\partial T^{\prime}}{\partial t^{\prime}}=k \frac{\partial^{2} T^{\prime}}{\partial y^{2}}-16 a \sigma T_{\infty}^{\prime 3}\left(T^{\prime}-T_{\infty}^{\prime}\right)
$$

On introducing the following non-dimensional quantities

$$
\begin{aligned}
& X=\frac{x u_{0}}{v}, \quad Y=\frac{y u_{0}}{v}, \quad U=\frac{u}{u_{0}}, \quad V=\frac{v}{u_{0}}, \quad t=\frac{t^{\prime} u_{0}^{2}}{v}, \\
& T=\frac{T^{\prime}-T_{\infty}^{\prime}}{T_{w}^{\prime}-T_{\infty}^{\prime}}, \quad \mathrm{Gr}=\frac{g \beta v\left(T_{w}^{\prime}-T_{\infty}^{\prime}\right)}{u_{0}^{3}}, \quad C=\frac{C^{\prime}-C_{\infty}^{\prime}}{C_{w}^{\prime}-C_{\infty}^{\prime}}, \quad \mathrm{Gc}=\frac{v g \beta^{*}\left(C_{w}^{\prime}-C_{\infty}^{\prime}\right)}{u_{0}^{3}}, \\
& \operatorname{Pr}=\frac{v}{\alpha}, \quad \mathrm{Sc}=\frac{v}{D}, \quad R=\frac{16 a^{*} v^{2} \sigma T_{\infty}^{3}}{k u_{0}^{2}}, \quad M=\frac{\sigma B_{0}^{2} v}{\rho u_{0}^{2}} .
\end{aligned}
$$

Equations (2.1) to (2.3) are reduced to the following non-dimensional form

$$
\begin{aligned}
& \frac{\partial U}{\partial t}=\operatorname{Gr} T+\operatorname{Gc} C+\frac{\partial^{2} U}{\partial Y^{2}}-M U, \\
& \frac{\partial T}{\partial t}=\frac{1}{\operatorname{Pr}} \frac{\partial^{2} T}{\partial Y^{2}}-\frac{R}{\operatorname{Pr}} T,
\end{aligned}
$$




$$
\frac{\partial C}{\partial t}=\frac{1}{\operatorname{Sc}} \frac{\partial^{2} C}{\partial Y^{2}}
$$

The corresponding initial and boundary conditions in non-dimensional quantities are

$$
\begin{aligned}
& t \leq 0: \quad U=0, \quad T=0, \quad C=0, \\
& t>0: \quad \quad \quad \quad T=1, \quad C=1 \quad \text { at } \quad Y=0 \text {, } \\
& U=0, \quad T=0, \quad C=0 \quad \text { at } \quad X=0 \text {, } \\
& U \rightarrow 0, \quad T \rightarrow 0, \quad C \rightarrow 0 \quad \text { as } \quad Y \rightarrow \infty \text {. }
\end{aligned}
$$

\section{Numerical technique}

In order to solve the unsteady, non-linear coupled Eqs (2.9) to (2.11) under the conditions (2.12), an implicit finite difference scheme of Crank-Nicolson type has been employed. The finite difference equations corresponding to Eqs (2.9) to (2.11) are as follows

$$
\begin{aligned}
& \frac{\left[U_{i, j}^{n+1}-U_{i, j}^{n}\right]}{\Delta t}=\frac{\mathrm{Gr}}{2}\left[T_{i, j}^{n+1}+T_{i, j}^{n}\right]+\frac{\mathrm{Gc}}{2}\left[C_{i, j}^{n+1}+C_{i, j}^{n}\right]-\frac{M}{2}\left[U_{i, j}^{n+1}+U_{i, j}^{n}\right]+ \\
& +\frac{\left[U_{i, j-1}^{n+1}-2 U_{i, j}^{n+1}+U_{i, j+1}^{n+1}+U_{i, j-1}^{n}-2 U_{i, j}^{n}+U_{i, j+1}^{n}\right]}{2(\Delta Y)^{2}}, \\
& \frac{\left[T_{i, j}^{n+1}-T_{i, j}^{n}\right]}{\Delta t}=\frac{1}{\operatorname{Pr}} \frac{\left[T_{i, j-1}^{n+1}-2 T_{i, j}^{n+1}+T_{i, j+1}^{n+1}+T_{i, j-1}^{n}-2 T_{i, j}^{n}+T_{i, j+1}^{n}\right]}{2(\Delta Y)^{2}}-\frac{\left(T_{i, j}^{n+1}+T_{i, j}^{n}\right)}{2 \operatorname{Pr}}, \\
& \frac{\left[C_{i, j}^{n+1}-C_{i, j}^{n}\right]}{\Delta t}=\frac{1}{\mathrm{Sc}} \frac{\left[C_{i, j-1}^{n+1}-2 C_{i, j}^{n+1}+C_{i, j+1}^{n+1}+C_{i, j-1}^{n}-2 C_{i, j}^{n}+C_{i, j+1}^{n}\right]}{2(\Delta Y)^{2}} .
\end{aligned}
$$

Here the region of integration is considered as a rectangle with sides $X_{\max }(=1)$ and $Y_{\max }(=14)$, where $Y_{\max }$ corresponds to $Y=\infty$ which lies very well outside both the momentum and energy boundary layers. The maximum of $Y$ was chosen as 14 after some preliminary investigations so that the last two of the boundary conditions (2.12) are satisfied with in the tolerance limit $10^{-5}$.

After experimenting with a few sets of mesh sizes they have been fixed at the level $\Delta X=0.05, \Delta Y$ $=0.25$, with time step $\Delta t=0.01$. In this case, the spatial mesh sizes are reduced by $50 \%$ in one direction, and later in both directions, and the results are compared. It is observed that when the mesh size is reduced by $50 \%$ in the $Y$-direction, the results differ in the fifth decimal place while the mesh sizes are reduced by $50 \%$ in $X$-direction or in both directions, the results are comparable to three decimal places. Hence, the above mesh sizes have been considered as appropriate for calculations. The coefficients $U_{i, j}^{n}$ appearing in the finite difference equation are treated as constants at any one time step. Here $i$-designates the grid point along the $X$ - direction, $j$ along the $Y$ - direction and $k$ to the $t$-time. The values of $U$ and $T$ are known at all grid points at $t$ $=0$ from the initial conditions.

The computations of $U, T$ and $C$ at time level $(n+1)$ using the values at previous time level $(n)$ are carried out as follows: the finite-difference Eq.(3.3) at every internal nodal point on a particular $i$-level constitutes a tridiagonal system of equations. Such a system of equations is solved by using the Thomas 
algorithm as discussed in Carnahan et al. (1969). Thus, the values of $C$ are found at every nodal point for a particular $i$ at $(n+1)^{\text {th }}$ time level. Similarly, the values of $T$ are calculated from Eq.(3.2) .Using the values of $C$ and $T$ at $(n+1)^{\text {th }}$ time level in Eq.(3.1), the values of $U$ at $(n+1)^{t h}$ time level are found in a similar manner. Thus, the values of $C, T$ and $U$ are known on a particular $i$-level. This process is repeated for various $i$-levels. Thus the values of $C, T$ and $U$ are known at all grid points in the rectangle region at $(n+1)^{\text {th }}$ time level.

\section{Discussion of results}

The velocity profiles for different values of the magnetic field parameter are shown in Fig.1. The velocity profiles presented are those at $X=1.0$. It is observed that for $M=0,2,5,10, R=2, \mathrm{Gr}=2, \mathrm{Gc}=5$, $\operatorname{Pr}=0.71$ and $\mathrm{Sc}=0.6$, the velocity decreases in the presence of the magnetic field than in its absence. This shows that the increase in the magnetic field parameter leads to a fall in the velocity. This agrees with the expectations, since the magnetic field exerts a retarding force on the free convective flow.

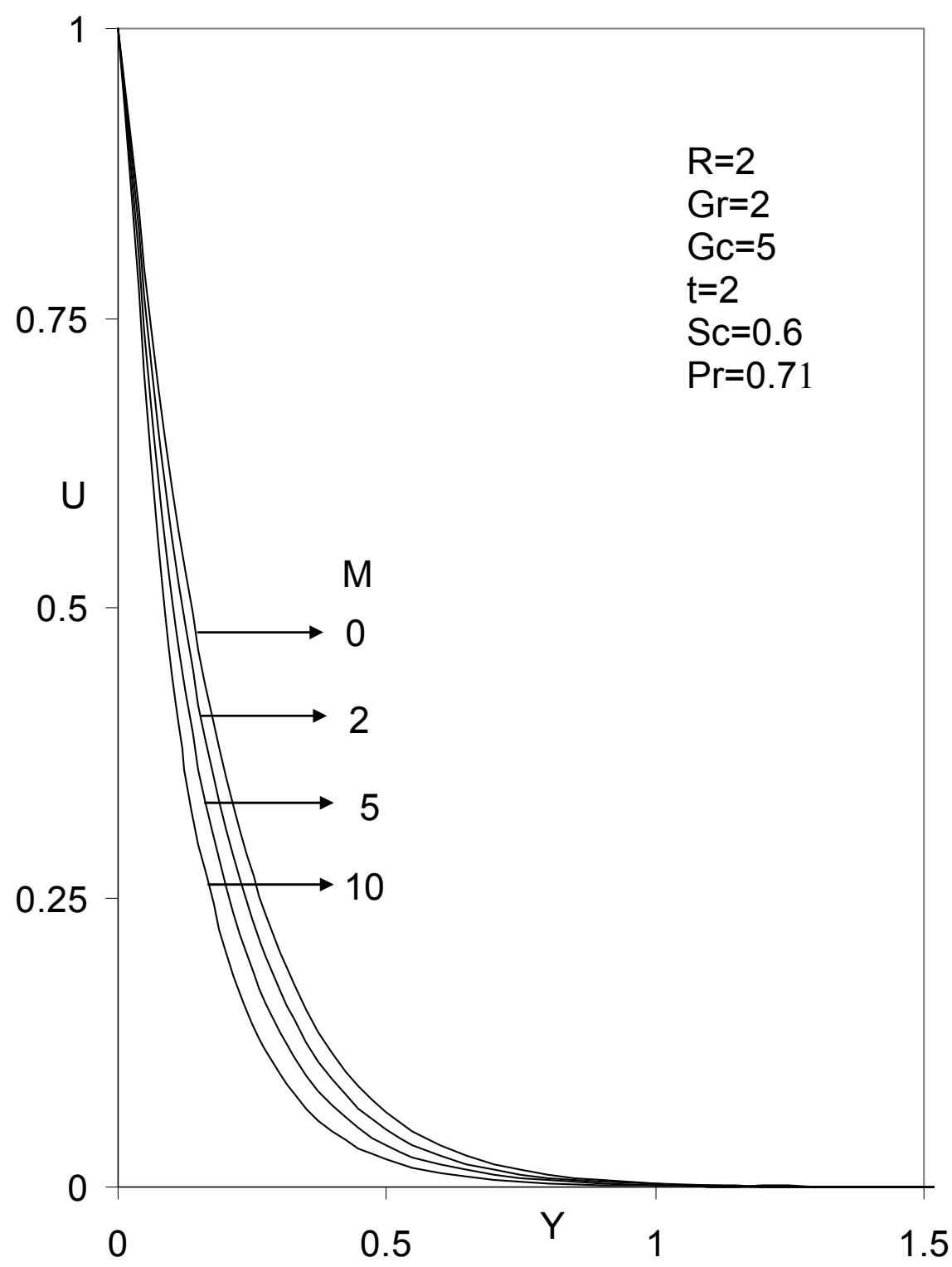

Fig.1. Velocity profiles for different values of $M$. 
The effect of velocity for different values of the radiation parameter $(R=0,2,5), M=2, \mathrm{Gr}=2$, $\mathrm{Gc}=5, \mathrm{Pr}=0.71$ and $\mathrm{Sc}=0.6$ are shown in Fig.2. It is observed that the velocity increases with decreasing the radiation parameter. This shows that velocity decreases in the presence of high thermal radiation.

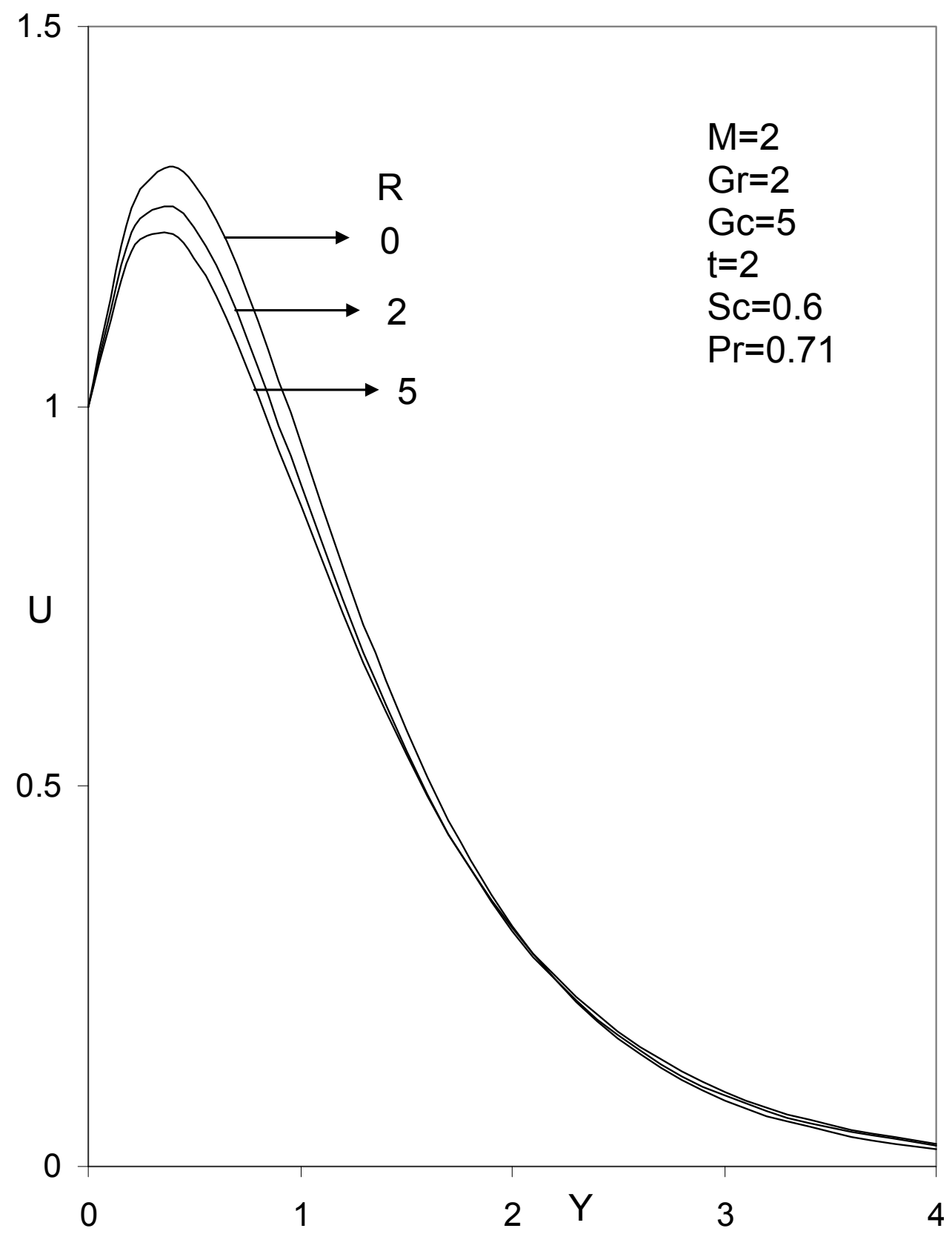

Fig.2. Velocity profiles for different values of $R$. 
In Fig.3, the velocity profiles for different values of the thermal Grashof number and mass Grashof number are shown graphically. This shows that the velocity increases with increasing the thermal Grashof number or mass Grashof number. As the thermal Grashof number or mass Grashof number increase, the buoyancy effect becomes more significant, as expected, it implies that more fluid is entrained from the free stream due to the strong buoyancy effects as Gr or Gc increases.

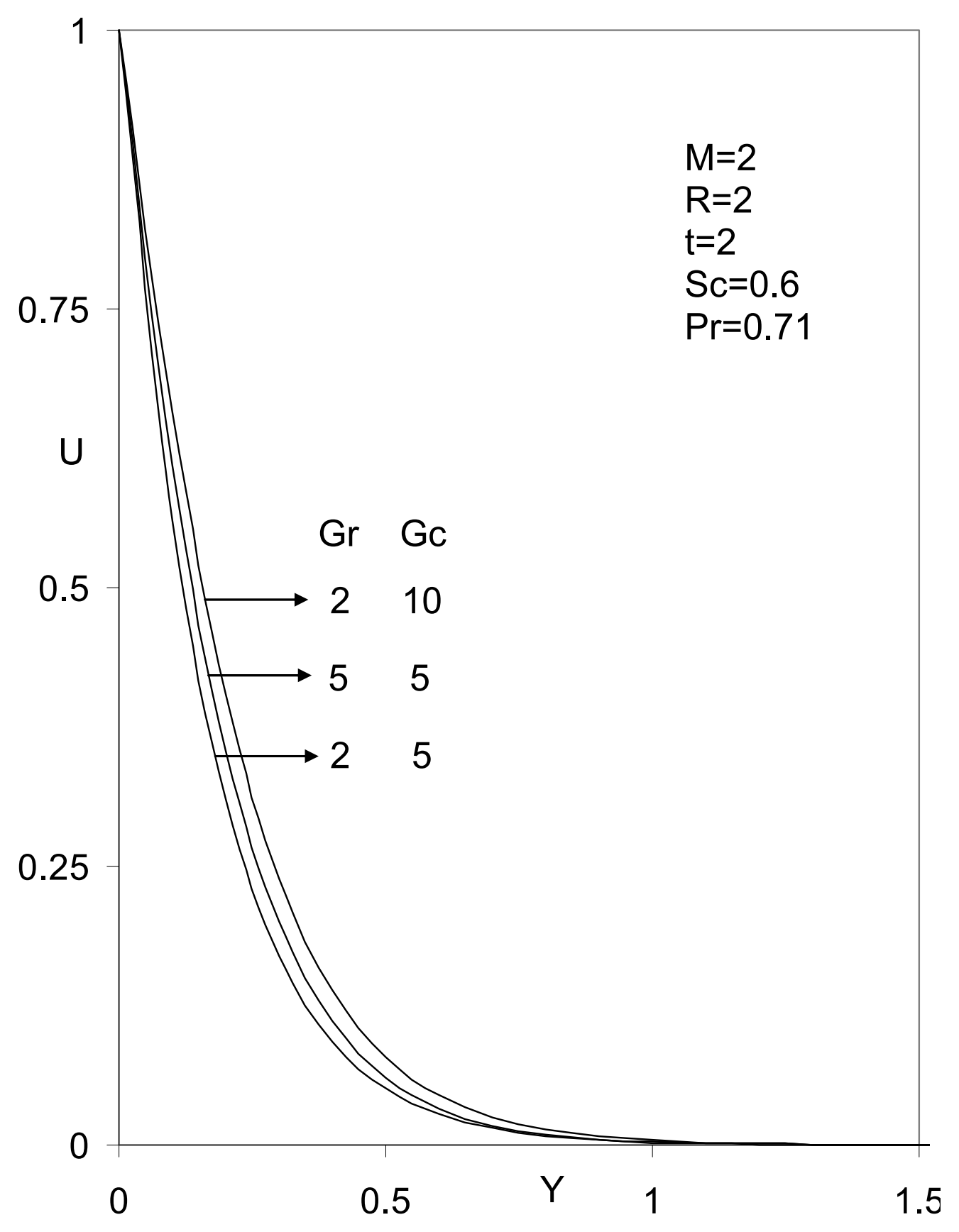

Fig.3. Velocity profiles for different values of Gr and Gc. 
The temperature profiles for different values of the thermal radiation parameter are shown in Fig.4. It is observed that the temperature increases with decreasing $R$. This shows that the buoyancy effect on the temperature distribution is very significant in air $(\mathrm{Pr}=0.71)$. It is known that the radiation parameter and Prandtl number plays an important role in flow phenomena because it is a measure of the relative magnitude of viscous boundary layer thickness to the thermal boundary layer thickness.

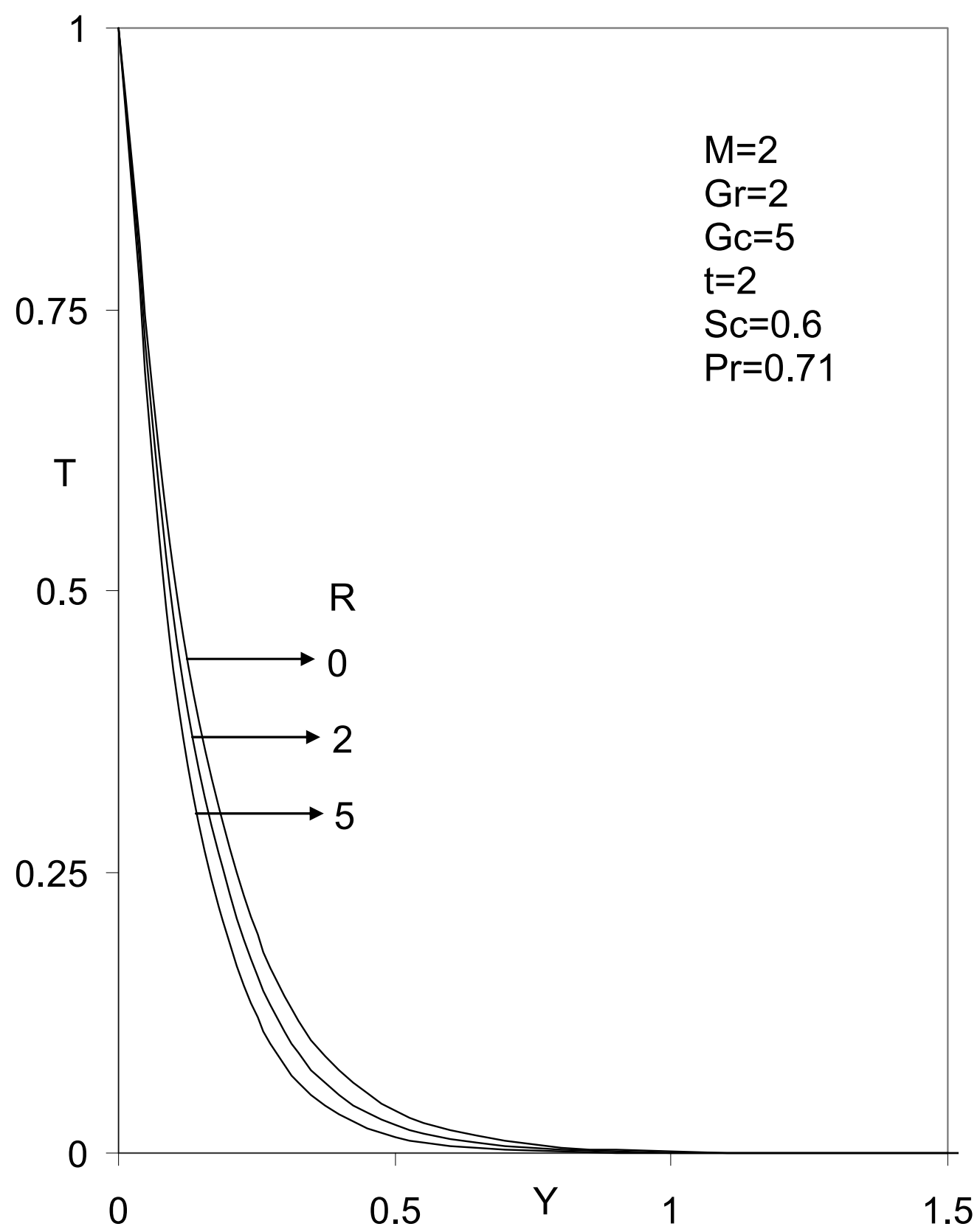

Fig.4. Temperature profiles for different values of $R$. 


\section{Conclusions}

A finite difference study has been carried out for an unsteady hydromagnetic flow past an impulsively started infinite vertical plate in the presence of thermal radiation. The dimensionless governing equations are solved by an implicit scheme of Crank-Nicolson type. The effects of velocity and temperature for different parameter are studied. It is observed that the velocity decreases in the presence of thermal radiation or the magnetic field.

\section{Nomenclature}

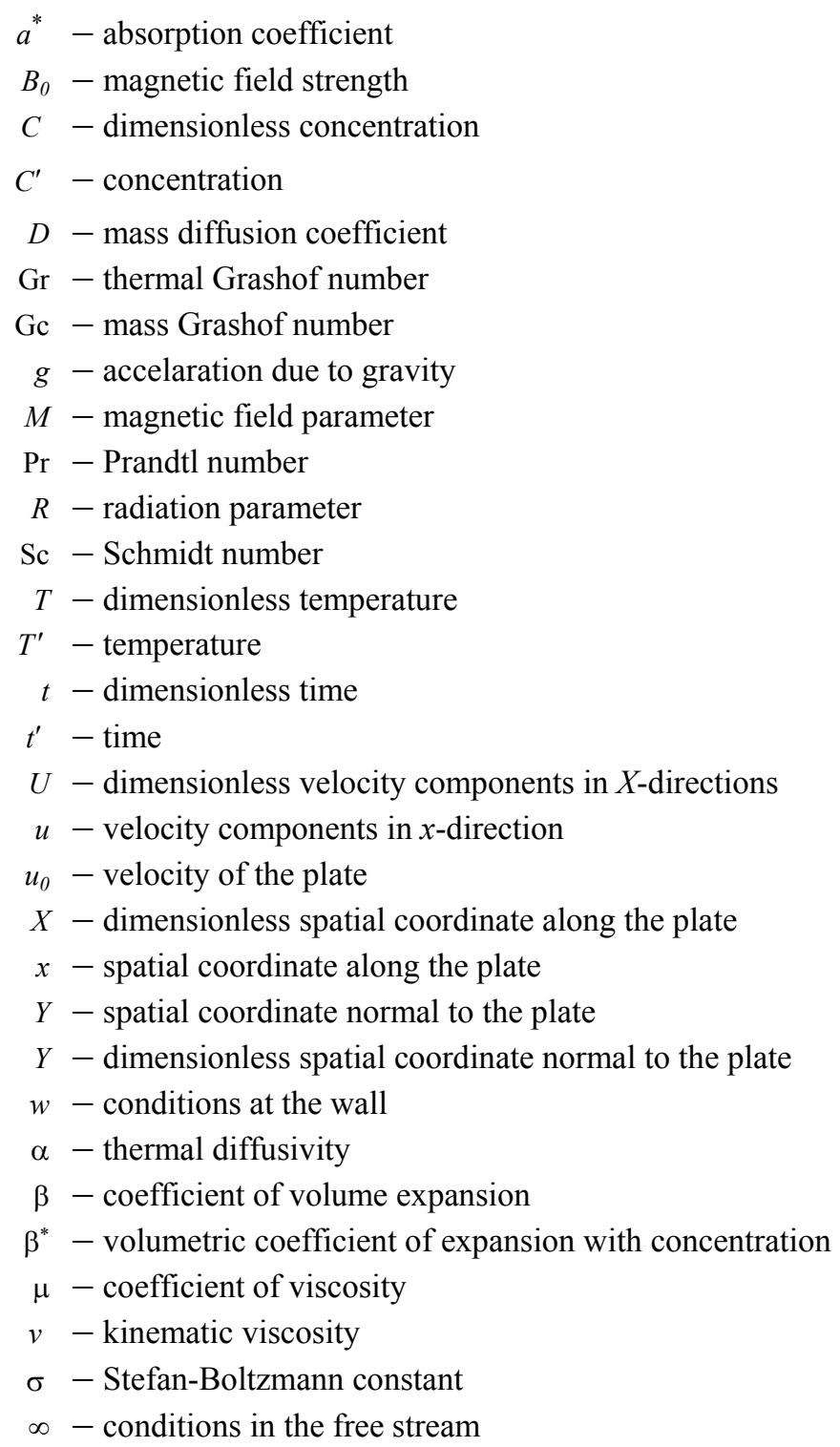

\section{References}

Carnahan B., Luther H.A. and Wilkes J.O.(1969): Applied Numerical Methods. - New York: John Wiley and Sons.

England W.G. and Emery A.F. (1969): Thermal radiation effects on the laminar free convection boundary layer of an absorbing gas. - J. Heat Transfer, vol.91, pp.37-44. 
Hossian M.A. and Takhar H.S. (1996): Radiation effect on mixed convection along a vertical plate with uniform surface temperature - Heat and Mass Transfer, vol.31, pp.243-248.

Kumari M. and Nath G. (1999): Development of two-dimensional boundary layer with an applied magnetic field due to an impulsive motion. - Indian J. of Pure and Applied Mathematics, vol.30, pp.695-708.

Raptis A. and Perdikis C. (1999): Radiation and free convection flow past a moving plate. - Int. J. App. Mech. and Engg., vol. 4, pp.817-821.

Raptis A. and Perdikis C. (2003): Thermal radiation of an optically thin gray gas. - Int. J. App. Mech. and Engg., vol. 8, pp.131-134.

Sakiadis B. C. (1961): Boundary layer behaviour on continuous solid surfaces: II.Boundary layer on a continuous flat surfaces. - AIChE Journal, vol.7, pp.221-225.

Soundalgekar V.M. (1979): Effects of mass transfer and free convection currents on the flow past an impulsively started vertical plate. - ASME Journal of Applied Mechanics, vol.46, pp.757-760.

Soundalgekar V.M., Gupta S.K. and Aranake R.N. (1979): Free convection effects on MHD Stokes problem for a vertical plate. - Nuclear Engg. Des., vol.51, pp.403-407.

Received: February 12, 2013

Revised: August 13, 2013 